\title{
Diacronie
}

Studi di Storia Contemporanea

$\mathrm{N}^{\circ} 36,4 \mid 2018$

Viaggi e turismo nell'Europa del Novecento

\section{Nota introduttiva n. 36 - dicembre 2018}

\section{Elisa Tizzoni}

\section{(2) OpenEdition}

\section{Journals}

\section{Edizione digitale}

URL: https://journals.openedition.org/diacronie/9729

DOI: 10.4000/diacronie.9729

ISSN: 2038-0925

\section{Editore}

Association culturelle Diacronie

\section{Notizia bibliografica digitale}

Elisa Tizzoni, «Nota introduttiva n. 36 - dicembre 2018», Diacronie [Online], № 36, 4 | 2018, online dal 29 décembre 2018, consultato il 17 février 2022. URL: http://journals.openedition.org/diacronie/9729 ; DOI: https://doi.org/10.4000/diacronie.9729

Questo documento è stato generato automaticamente il 17 février 2022.

Creative Commons License 
Nota introduttiva n. 36 - dicembre 2018

Elisa Tizzoni

Viaggi e turismo nell'Europa del Novecento 
1 Il numero 36 di Diacronie è dedicato al tema Viaggi e turismo nell'Europa del Novecento e si inserisce pertanto in uno dei più vivaci campi di indagine della storiografia contemporanea.

2 Mentre la pratica del viaggio rappresenta un ambito di ricerca consolidato ${ }^{1}$, la storia del turismo è emersa come filone autonomo solo in epoche relativamente recenti: se si eccettuano alcune ricerche pionieristiche condotte da Marc Boyer ${ }^{2}$ sin dagli anni Sessanta, le opere più significative nella letteratura internazionale datano al periodo compreso tra la fine degli anni Novanta e di primi anni Duemila ${ }^{3}$.

3 L'invito più deciso (e più affascinante) ad indagare storicamente il turismo nelle sue diverse manifestazioni proviene da uno

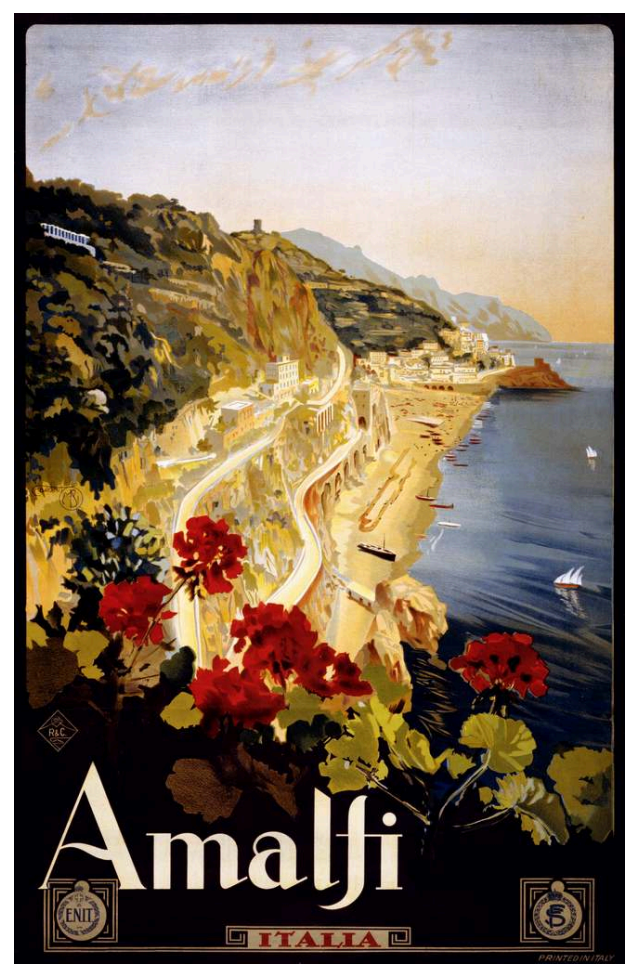
dei massimi esperti di questo tema, John K. Walton, secondo il quale «tourism celebrates, manipulates, appropriates, challenges and displaces distinctive aspects of local environments and cultures in a Protean variety of ways, generating exciting grounded case studies for historians to work on» ${ }^{4}$.

4 L'analisi storica, dunque, rivela come il turismo sia un fenomeno ben più complesso di quanto non faccia intuire la sintetica definizione di "turismo" fornita dalla World tourism organisation, ${ }^{5}$ che consente di gettare nuova luce su complessi nodi storiografici di particolare rilievo per lo studio dell'epoca contemporanea. L'evoluzione dei modelli di consumo nel Mondo occidentale, l'accresciuto ruolo del potere pubblico in Europa sino alla diffusione del welfare state, i risvolti a lungo termine del processo di decolonizzazione, l'impatto della guerra fredda sulle abitudini di consumo, sono alcune delle questioni nelle quali il turismo ha giocato un ruolo di rilievo e che possono essere meglio comprese prendendo in considerazione la pratica del viaggio nella sua dimensione individuale e, soprattutto, collettiva.

5 Dal punto di vista metodologico, accanto ai più consueti approcci della storia economica e della storia dei consumi più recentemente altri indirizzi di ricerca sono stati applicati alla storia del viaggio e del turismo, tra i quali la storia culturale ${ }^{6}$, la storia politica, con particolare riferimento ai regimi totalitari ${ }^{7}$ e alla stagione della Guerra Fredda $^{8}$, la storia ambientale ${ }^{9}$, la labour history ${ }^{10}$.

6 A fronte della ricchezza di temi ed approcci di indagine, potremmo domandarci cos'è dunque oggi la storia del turismo, se essa rappresenti una corrente storiografica autonoma o, piuttosto, una prospettiva trasversale rispetto a diverse discipline.

7 Una definizione "astratta" delle finalità e delle caratteristiche della storia del turismo, tuttavia, rischierebbe di non cogliere la vitalità di questo filone, capace di rielaborare efficacemente suggerimenti metodologici provenienti dalle più avanzate punte della 
storiografia contemporanea e di rivolgere uno sguardo nuovo a temi già ampliamenti analizzati in lavori precedenti.

8 Non bisogna dimenticare, inoltre, che il viaggio e, soprattutto, il turismo condizionano profondamente le politiche pubbliche, le dinamiche sociali e i processi economici del mondo di oggi, pratiche che offrono potenzialità di crescita materiale e culturale non ancora esplorate del tutto, ma che possono anche minacciare gli equilibri dell'ambiente naturale ed antropico, sollecitando dunque gli storici ad un'approfondita analisi delle dinamiche che hanno condotto alla loro diffusione.

La comprensione delle radici storiche del turismo e dei cambiamenti maggiori che hanno interessato la pratica del viaggio in senso ampio rappresenta dunque un'avvincente sfida per gli storici, una sfida che Diacronie ha voluto raccogliere provando ad esplorare alcune delle principali direttrici di ricerca in questo campo.

10 I contributi presenti in questo numero fanno tesoro del dibattito storiografico precedente e, pure, propongono angoli di visuale nuovi e prendono in esame temi sinora oggetto di scarsa attenzione, adottando, nel contempo, una prospettiva fortemente interdisciplinare

11 La scelta dei limiti geografici e temporali entro i quali collocare i saggi non è stata dettata solamente da esigenze di coerenza tematica, ma discende, in parte, dalla considerazione delle peculiarità del contesto europeo e dell'accelerazione impressa all'evoluzione delle pratiche di viaggio nel Novecento.

Da quanto anticipato risulta evidente che nel Ventesimo secolo, termine cronologico di riferimento per il presente numero, il turismo ha assunto una dimensione propria e ben distinta da precedenti pratiche ludiche, mentre il viaggio si è caricato di significati nuovi rispondenti ai mutati scenari geopolitici, economici, sociali.

Sottolineiamo, inoltre, che le pratiche di viaggio e turismo verso destinazioni del Vecchio Continente hanno assunto un carattere sempre più "europeo", rappresentando un tassello importante dell'intricato processo di identity building richiesto dalla crescente integrazione europea.

Questo numero, dunque, si concentra sul turismo in Europa evidenziando la presenza di flussi di carattere globale, consolidatisi nel corso delle Trente Glorieuses dei consumi di massa. Nel contempo, i saggi selezionati non trascurano gli aspetti "globali" dei processi economici e sociali sviluppatisi nell'Europa del Ventesimo secolo, rileggendo alcune tendenze del turismo internazionale nella prospettiva dei colonial e post-colonial studies.

15 Coerentemente con l'interesse che Diacronie ha sempre dimostrato verso le contaminazioni tra discipline e metodologie differenti, i contributi integrano gli strumenti della ricerca storica con quelli messi a disposizione da sociologia, economia, geografia, applicandoli allo studio di una vasta gamma di fonti che comprende, accanto a quelle documentarie, anche fonti qualitative, visuali, orali.

16 Al fine di facilitare la lettura complessiva di un corposo numero di saggi, estremamente diversi per approccio e ambito di indagine, abbiamo individuato tre sezioni tematiche, dedicate rispettivamente al percorso che ha condotto dal viaggio elitario al turismo di massa, agli usi politici del turismo e al turismo in Europa in prospettiva globale.

Nella prima sezione, Martine Tabeaud e Alexis Metzger analizzano un corpus di immagini prodotte per promuovere il turismo ferroviario in Svizzera, evidenziando efficacemente come, nel periodo di tempo compreso tra la belle époque e l'esplosione del 
consumo di massa, sia cresciuta la consapevolezza delle potenzialità (ma anche delle fragilità) dell'ambiente naturale alpino. Spostando l'attenzione verso l'area mediterranea, Javier Márquez Quevedo ricostruisce le principali dinamiche economiche e politiche innescate dal boom del turismo balneare nelle Isole Canarie a partire dagli anni Sessanta fino alla metà del decennio successivo, mentre Bertrand Réau rilegge l'esperienza dei primi Club Mediterranée da una prospettiva socio-storica, individuando le peculiarità di questa nuova forma di condivisione dell'esperienza di viaggio.

Un altro tema classico della storia del turismo, l'evoluzione del termalismo contemporaneo, viene esaminato da una nuova angolazione da parte di Riccardo Semeraro e Maria Paola Pasini, che si concentrano sul ricco corpus archivistico prodotto dalla S.A. Terme e Grandi Alberghi di Sirmione nel secondo dopoguerra.

La prima sessione si chiude con un saggio particolarmente originale per l'oggetto e le categorie di analisi applicate, nel quale Luciano Maffi e Martino Lorenzo Fagnani esplorano il nesso tra turismo, disabilità e inclusione focalizzandosi sull'esperienza dell'Istituto dei ciechi di Milano.

Nella seconda sezione l'utilizzo politico del turismo viene collocato in due scenari, quello delle dittature mediterranee mediterranei e quello della Guerra fredda.

Nel primo caso Christophe Poupault si concentra sulla non sempre facile coesistenza tra obiettivi di sviluppo del turismo e ambizioni totalitarie nelle politiche del fascismo italiano, mentre Carla Ribeiro rivolge l'attenzione all'utilizzo del turismo come strumento per accrescere il consenso verso il regime estadonovista portoghese.

Le nuove connotazioni politiche assunte dai viaggi di scambio e dal turismo sociale nel contesto del mondo bipolare sono indagate da William Richier, prendendo in esame $\mathrm{i}$ viaggi organizzati nella Repubblica Democratica Tedesca dall'Associazione francese legata al Pcf Échanges Franco-Allemand, e da Mario Giulio Salzano, che riflette sui complessi significati assunti dal Hadž (Hajj) (il "viaggio della fede") nella Jugoslavia socialista tra il 1949 e il 1961.

La terza sezione adotta una prospettiva globale attraverso una triplice visuale, che prende in esame i rapporti dei paesi europei con le aree coloniali e postcoloniali, il significato della "destinazione Europa" per diverse categorie di viaggiatori nordamericani e le connessioni multiple che si stabiliscono a livello internazionale nell'ambito delle pratiche turistiche.

24 Nel primo contributo Sihem Bella analizza le rappresentazioni della città di Algeri all'interno delle principali guide turistiche francesi pubblicate nel corso del Novecento, evidenziando le complesse eredità del passato coloniale, mentre Katherine Cartwright indaga la pratica del viaggio in Europa da parte delle giovani turiste nordamericane nel periodo interbellico, pervenendo a interessanti conclusioni sull'identità di genere e sulla youth culture dell'epoca. La sezione si chiude con il saggio di Francesco Fusi, che si interroga sui significati delle visits home dei soldati italo-americani durante la Campagna d'Italia, sviluppando alcuni dei più interessanti suggerimenti di ricerca offerti dai diaspora studies, e con la ricerca di Gloria Priego-de-Montiano, nella quale si individuano inaspettati parallelismi a lungo raggio tra Europa Settentrionale e Meridionale nel settore del turismo rurale.

Chiude questo numero il consueto spazio dedicato alle recensioni (IV. Recensioni), con un'attenzione particolare al tema del terrorismo. 
La curatela del monografico è di Elisa Tizzoni, il coordinamento redazionale è di Deborah Paci.

Buona lettura,

Elisa Tizzoni

\section{NOTE}

1. Nell'impossibilità di fornire puntuali indicazioni sulla sterminata bibliografia in materia, ci limitiamo a segnalare: BERTRAND, Gilles (dir.), La culture du voyage. Pratiques et discours de la Renaissance à l'aube du XXe siècle, Paris, L'Harmattan, 2004.

2. BOYER, Marc, «Hyeres, Station d'hivernants au XIXe siècle», in Provence Historique, 12, 1962, pp. 139-165.

3. WILTHEY, Lynne, Grand tours and Cooks' tours: a history of leisure travel, 1750-1915, New York, W. Morrow, 1997; FURLOUGH, Ellen, «Making Mass Vacations: Tourism and Consumer Culture in France, 1930s to 1970s», in Comparative Studies in Society and History, 40/2 (1998), pp. 247-286; CORMACK, Bill, A History of Holidays 1812-1990, London, Routledge, 1998; LÖFGREN, Orvar, On Holiday. A History of Vacationing, Berkeley, University of California Press, 1999; BARANOWSKI, Shelley, FURLOUGH, Ellen, (Eds), Being Elsewhere: Tourism, Consumer Culture, and Identity in Modern Europe and North America, Ann Arbor, University of Michigan Press, 2001; BATTILANI, Patrizia, Vacanze di pochi, vacanze di tutti. L'evoluzione del turismo europeo, Bologna, Il Mulino, 2001.

4. WALTON, John K., «Welcome to the Journal of Tourism History», in Journal of Tourism History, 1 (2009), p. 2.

5. «Tourism comprises the activities of persons traveling to and staying in places outside their usual environment for not more than one consecutive year for leisure, business and other purposes».

6. BARANOWSKI, Shelley, FURLOUGH, Ellen, op. cit.

7. BARANOWSKI, Shelley, Strength through Joy: Consumerism and Mass Tourism in the Third Reich, Cambridge, Cambridge University Press, 2004; SYRJÄMAA, Taina, Visitez l'Italie. Italian State Tourist Propaganda Abroad 1919-1943. Administrative Structure and Practical Realization, Turku, Turun Yliopisto, 1997; ECKERT, Astrid M., «'Greetings from the Zonal Border’ Tourism to the Iron Curtain in West Germany», in Zeithistorische Forschung/Studies in Contemporary History, 8 (2011), 1, pp. 9-36.

8. ENDY, Christopher, Cold War Holidays: American Tourism in France, Chapel Hill, University of North Carolina Press, 2004.

9. MORANDA, Scott, «The emergence of an environmental history of tourism», in Journal of Tourism History, 7/3 (2015), pp. 1-22.

10. BARTON, Susan, Working-Class Organisations and Popular Tourism, 1840-1970, Manchester, Manchester University Press, 2005; PATTIEU, Sylvain, Tourisme et travail, De l'éducation populaire au secteur marchand (1945-1985), Paris, Presses de Sciences Po, 2009. 


\section{AUTORE}

\section{ELISA TIZZONI}

Elisa Tizzoni (La Spezia, 1983), PhD in Storia contemporanea, ha svolto incarichi di ricerca ed insegnamento presso le Università di Firenze, Pisa, Nizza, Salisburgo e presso altre istituzioni culturali; nel 2017 ha ottenuto una Postgraduate Vibeke Sørensen Grant presso l'Historical Archives of the European Union a Firenze. Attualmente è visiting fellow presso il Department of History and Civilization dell'Istituto Universitario Europeo di Firenze e Cultore della Materia presso il Dipartimento di Storia e Civiltà dell'Università di Pisa.

URL: < http://www.studistorici.com/2019/03/07/elisa-tizzoni/ > 